\title{
Paracoccidioidomicosis: definición de las áreas endémicas de Colombia
}

\author{
Edilma Torrado ${ }^{1}$, Elizabeth Castañeda ${ }^{1}$, Fernando de la $\mathrm{Hoz}^{2}$, Angela Restrepo ${ }^{3}$ \\ ${ }_{1}^{1}$ Grupo de Microbiología, Instituto Nacional de Salud, Bogotá, D.C., Colombia. \\ ${ }^{2}$ Centro Control de Enfermedades, Instituto Nacional de Salud, Bogotá, D.C., Colombia. \\ ${ }^{3}$ Corporación para Investigaciones Biológicas, Medellín, Colombia.
}

Una enfermedad endémica es aquélla que se presenta constantemente en un área geográfica o en un grupo de población; la definición de endemia abarca, también, la prevalencia usual de una enfermedad en un área o grupo determinado. El objetivo de este estudio fue el establecer la endemicidad de la paracoccidioidomicosis en los 32 departamentos colombianos y asociarla con algunas de las características geográficas correspondientes a estas áreas. Se realizó un estudio retrospectivo de 1949 a 1999, para el cual sirvieron como fuentes de información cuatro laboratorios de micología, dos servicios de patología y, además, la literatura colombiana. Las principales variables consignadas fueron el año de diagnóstico y la procedencia de los pacientes. Se calcularon las tasas de incidencia por departamento con base en los censos de 1973 , 1985, 1993 y en sus proyecciones para 1990 y 1995. La definición de áreas endémicas estuvo basada en la distribución de los casos por lugar y tiempo, usando como medidas estadísticas la media y los cuartiles. Estas medidas se aplicaron al total de casos diagnosticados por departamento y a su diagnóstico anual. Se analizaron 1.191 casos en un período de 50 años. Con base en el número total de casos diagnosticados por departamento, 9 de ellos se clasificaron como endémicos ya que informaron más de 32 casos por año; 8 correspondieron a la región andina y 1 a la Sierra Nevada de Santa Marta. Dieciocho departamentos se catalogaron como regiones de baja endemicidad (2-27 casos) y los 5 restantes, como no endémicos ( 1 caso). Entre 1980-1998 y con base en el diagnóstico anual de casos, se encontraron 8 departamentos que habían diagnosticado la enfermedad por períodos de 13 a 19 años, lo que permitió clasificarlos como endémicos; adicionalmente, 15 fueron considerados de baja endemicidad, con base en un período de diagnóstico de 2 a 12 años; los restantes 10 fueron clasificados como no endémicos por haber informado la entidad solamente en un 1 año. Ambos análisis coincidieron en catalogar 8 de los departamentos como endémicos. La incidencia nacional más alta se obtuvo en 1980 con 2,4 pacientes/1'000.000 habitantes. La tasa de incidencia de la enfermedad no constituyó un parámetro adecuado para la definición de endemicidad. Estos hallazgos corroboran que la distribución de la paracoccidioidomicosis en Colombia es heterogénea y, además, ofrecen una alternativa para determinar el hábitat del agente etiológico por medio de estudios de campo.

Palabras clave: paracoccidioidomicosis, endemicidad, casos colombianos, áreas endémicas.

\section{Definition of paracoccidioidomycosis endemic areas in Colombia}

An endemic disease is one that is constantly present within a geographical area or population group; the definition of endemia also encompasses the usual prevalence of a disease within a defined area or a determined group. The aim of this work was to establish the endemicity of paracoccidioidomycosis in the 32 Colombian departments (political divisions) and to associate it with certain geographical characteristics such as altitude. A retrospective study was carried out from 1949 to 1999. The data collected referred to the year of diagnosis and patients' residence locality, while the information was derived from four different mycology laboratories, two pathology services and the Colombian publications on the disease. The incidence rate for department was obtained from three individual censuses, 1973, 1985 and 1993, as well as from projections for 1990 and 1995. Definition of endemic areas was based on the distribution of case frequencies by 
place and time. Statistical measurements used were the median and the quartiles. In the 50year period 1,191 cases had been recorded and were analyzed here. Based on the total number of cases diagnosed for department, 9 were classified as endemic, having reported more than 32 cases per year; 8 of them belonged to the Andean region and 1 to the Sierra Nevada of Santa Marta. Eighteen departments were catalogued as low endemic regions, as they had reported only 2-27 cases, while the remaining 5 reported 1 or no cases and were considered non-endemic. In the period from 1980 to 1998,8 departments diagnosed the disease regularly for 13-19 years, and they were classified as endemic. Fifteen departments were considered of low endemicity as they informed the mycosis for only 2-12 years, while 10 were regarded as non-endemic because they informed cases for less than 1 year. Both analyses agreed in cataloguing 8 departments as endemic. The higher national incidence was obtained in 1980 with 2.4 patients/1'000,000 inhabitants. The incidence rate of the disease was found to be an inadequate parameter to define endemicity. The findings corroborate that the distribution of paracoccidioidomycosis in Colombia is heterogeneous, and, additionally, offer an alternative way to study the habitat of the etiological agent.

Key words: paracoccidioidomycosis, endemicity, Colombian cases, endemic areas.

El estudio de la paracoccidioidomicosis se inició en Brasil, en 1908, cuando Lutz informó los dos primeros pacientes con una enfermedad mucocutánea crónica de etiología desconocida. Lutz reconoció que esta enfermedad era diferente de la blastomicosis, informada en Estados Unidos, y de la coccidioidomicosis, recién descrita por Posadas en la Argentina, y la clasificó como una 'nueva micosis pseudococcidioídica'. Lutz también logró aislar el agente etiológico y Splendore lo estudió en detalle; ambos demostraron que era un hongo dimórfico que presentaba la forma de levadura in vivo y la forma micelial in vitro $(1,2)$.

En 1930, Almeida aclaró la verdadera naturaleza de la enfermedad al estudiar su patogenia en modelos animales y determinó que el hongo era un nuevo agente, al cual denominó Paracoccidioides brasiliensis $(1,2)$. En 1933, se informó el primer caso fuera de Brasil, en Argentina, y posteriormente se describieron nuevos casos en Paraguay, Venezuela y Perú. En Colombia, el primer paciente fue diagnosticado en 1949 por Méndez Lemaitre en el Instituto Nacional de Radium (3).

Se sabe que la distribución geográfica de la paracoccidioidomicosis está limitada a Latinoamérica, desde México hasta Argentina. Brasil es el país con el mayor número de casos

Correspondencia: Elizabeth Castañeda ecastaneda@hemagogus.ins.gov.co

Recibido: 3/11/00; aceptado: 10/11/00 autóctonos, seguido a distancia por Venezuela y Colombia; la entidad es la micosis sistémica más importante de Latinoamérica (4).

Una enfermedad endémica es aquélla que se presenta constantemente en una determinada área geográfica o en un grupo definido de población; la definición de endemia abarca, también, la prevalencia usual de una enfermedad dentro de un área o grupo definidos (5). La mayoría de los estudios realizados sobre la endemicidad de la paracoccidioidomicosis se han realizado en Brasil, país donde la zona de mayor endemicidad corresponde al norte del estado de Rio Grande do Sul. Presenta una prevalencia de 6,6/100.000 hab en aquellas zonas donde los suelos son fértiles y las lluvias frecuentes todo el año; allí, predominan los bosques y la agricultura es una ocupación importante para los residentes $(4,6)$.

En la paracoccidioidomicosis, la delimitación de las áreas endémicas se ha basado solamente en el informe de casos clínicos, dado que no pueden emplearse otros métodos. Por ejemplo, el nicho ecológico de $P$. brasiliensis no ha sido establecido completamente debido a factores como la dificultad para aislar el hongo a partir del medio ambiente, el prolongado período de latencia de la enfermedad y los pocos informes sobre su presencia en animales (7). Esta dificultad llevó a Borelli a crear el término 'reservárea' para indicar el sitio particular donde el hongo vive en la naturaleza y el paciente adquiere la infección; en esta forma, Borelli diferenció la 'reservárea' del 'área 
endémica', la que corresponde al lugar donde se diagnostican y se informan los casos $(4,7)$.

El objetivo del presente trabajo fue tratar de establecer la endemicidad de la enfermedad en los 32 departamentos, con base en el número de casos clínicos de paracoccidioidomicosis informados en la literatura colombiana.

\section{Materiales y métodos}

\section{Tipo de estudio}

Se realizó un estudio retrospectivo que abarcó el período de 1949 a 1999 y que consistió en la consulta, análisis y recolección de datos sobre el año de diagnóstico y el departamento de procedencia de los pacientes con paracoccidioidomicosis.

\section{Fuente de información}

Esta estuvo centrada en cuatro laboratorios de micología, dos servicios de patología y las publicaciones colombianas sobre esta micosis (3,828). La definición de caso se aplicó a los individuos sintomáticos en los que se hubiera visualizado el hongo en exámenes directos o en biopsias, se hubiera recuperado $P$. brasiliensis en cultivo o en los que existiera evidencia serológica de paracoccidioidomicosis, esto es, demostración de anticuerpos contra el hongo por técnicas de inmunodifusión o fijación de complemento.

Con el objeto de no contabilizar el mismo paciente en más de una oportunidad, se tomaron ciertas precauciones, como constatar los nombres y apellidos en los registros disponibles en los centros de referencia o compararlos con aquéllos obtenidos de los laboratorios de patología que fueron consultados. Esta tarea se facilitó gracias a la preparación de una base de datos hecha por orden alfabético. En cuanto a los casos informados en las publicaciones, se tuvieron en cuenta las fechas cuando se había realizado el estudio y para una misma región; se aceptaron únicamente aquellos informes en los que tales fechas diferían claramente. Adicionalmente, se requería información sobre la procedencia de los pacientes.

\section{Análisis}

La definición de las áreas endémicas se basó en la distribución de frecuencias de los casos por lugar y tiempo, utilizando como medidas de posición la mediana y los cuartiles. Se emplearon dos criterios, el número de casos informados por departamento en el período de análisis (1949 a 1999) y el número de años en los cuales cada departamento había diagnosticado la enfermedad (1980-1999).

Para el primer análisis, se ordenó el número de casos por frecuencia y se calculó la mediana y los percentiles 25 y 75 . Se interpretaron como departamentos endémicos los que tenían casos por encima del percentil 75 y de baja endemicidad los que presentaron casos por encima del percentil 25. Para el análisis basado en el número de años de diagnóstico, se empleó la misma interpretación para la clasificación de endemia.

La determinación de las tasas de incidencia por departamento (por millón de habitantes) se realizó con base en los censos de 1973,1985 y 1993 , y en sus proyecciones para 1990 y 1995.

\section{Resultados}

A partir de las fuentes de información establecidas, se obtuvieron datos sobre 1.191 pacientes diagnosticados en el período de estudio, 983 procedentes de los laboratorios de micología y de los servicios de patología y 208 obtenidos de la búsqueda bibliográfica (cuadro 1). Del total de pacientes publicados, se excluyeron aquéllos en los cuales su procedencia no se había consignado (cuadro 2).

Con base en el número total de casos diagnosticados en los 32 departamentos, se obtuvo una mediana de 7 casos para cada uno de ellos y con base en esta mediana, los departamentos se dividieron en 4 categorías de acuerdo con el número de casos. En el cuartil 4, se agruparon nueve departamentos que tenían 320 más casos; en el cuartil 3, seis departamentos con 9 a 26 casos y en el cuartil 2, ocho departamentos con 3 a 7 casos. Con base en estos parámetros, se estableció que los primeros 9 departamentos eran endémicos, 14 eran de baja endemicidad y los 9 restantes, no endémicos (cuadro 3).

En un análisis independiente realizado en el período de 1980 a 1999 , se estudió el número de años en los cuales cada departamento había 
Cuadro 1. Número de casos de paracoccidioidomicosis registrados en Colombia (1949 a 1999).

\begin{tabular}{lrrr}
\hline Fuente & Años & $\mathrm{n}$ & Referencias \\
\hline Instituciones & & & \\
$\quad$ Corporación para Investigaciones Biológicas, Medellín & $1968-1999$ & 193 & 25,28 \\
Hospital San Vicente de Paúl, Medellín & $1956-1996$ & 65 & 24,26 \\
$\quad$ Universidad Industrial de Santander, Bucaramanga & $1954-1999$ & 197 & 5 \\
Hospital Universitario del Valle, Cali & $1990-1998$ & 31 & 21,27 \\
$\quad$ Instituto Nacional de Salud, Bogotá & $1953-1994$ & 492 & $3,8,10-20,22,23$ \\
$\quad$ Patología & $1977-1999$ & 208 & 1.191 \\
$\quad$ Microbiología & $1949-1992$ & & \\
Publicaciones & & & \\
Total & & & \\
\hline
\end{tabular}

Cuadro 2. Número de casos colombianos de paracoccidioidomicosis publicados (1949-1999) por año de diagnóstico, departamento de origen y referencia.

\begin{tabular}{|c|c|c|c|}
\hline $\begin{array}{l}\text { Año de } \\
\text { diagnóstico }\end{array}$ & $\begin{array}{c}\text { Casos } \\
n\end{array}$ & Procedencia (departamento) & Referencias \\
\hline $1948-1967$ & 373 & $\begin{array}{l}\text { Antioquia, Atlántico, Bolivar, Boyacá, Córdoba, } \\
\text { Cundinamarca, Caldas, Cauca, Chocó, Huila, } \\
\text { Quindío, Risaralda, Norte de Santander, } \\
\text { Santander, Tolima, Valle, Nariño, Caquetá, Meta }\end{array}$ & $\begin{array}{l}17(3,10 \\
12,13,18)\end{array}$ \\
\hline $1949-1950$ & 8 & $\begin{array}{l}\text { Caldas, Santander, Norte de Santander, } \\
\text { Quindío, Cundinamarca, Caquetá }\end{array}$ & 3 \\
\hline 1950 & 7 & Antioquia, Tolima & 15 \\
\hline 1953-1992 & 198 & Santander & 26 \\
\hline 1954 & 1 & Antioquia & 8 \\
\hline 1957 & 8 & Cauca, Meta, Santander, Boyacá, Magdalena, & 10 \\
\hline $1958-1972$ & 6 & Caquetá, Cundinamarca, Antioquia, Casanare, Santander & 19 \\
\hline $1959-1965$ & 39 & $\begin{array}{l}\text { Cundinamarca, Meta, Tolima, Santander, Cauca, Antioquia, } \\
\text { Huila, Boyacá, Chocó, Nariño, Valle, } \\
\text { Caldas }\end{array}$ & $\begin{array}{l}14 \\
1\end{array}$ \\
\hline 1963 & 1 & Bolivar & 11 \\
\hline $1964-1968$ & 39 & Antioquia & 18 \\
\hline 1965 & 16 & Valle & 12 \\
\hline 1965 & 3 & Antioquia & 13 \\
\hline 1967 & 20 & Antioquia, Valle, Chocó, Santander & 16 \\
\hline 1975 & 1 & Santander & 20 \\
\hline $1970-1987$ & 138 & Santander & 24 \\
\hline $1977-1980$ & 87 & $\begin{array}{l}\text { Cundinamarca, Santander, } \\
\text { Caldas, Boyacá, Meta, Tolima, } \\
\text { Magdalena, Caquetá, Valle, Huila, Norte de Santander, } \\
\text { Risaralda, Casanare, Arauca }\end{array}$ & 21 \\
\hline $1978-1990$ & 100 & Antioquia & 25 \\
\hline 1980 & 1 & Antioquia & 22 \\
\hline 1981-1992 & 333 & $\begin{array}{l}\text { Cundinamarca, Santander, } \\
\text { Caldas, Boyacá, Meta, Tolima, Magdalena, } \\
\text { Caquetá, Valle, Huila, Norte de Santander, } \\
\text { Risaralda, Casanare, Arauca, Cesar, Antioquia, } \\
\text { Nariño, Putumayo, Cauca, Vaupés, Guanía }\end{array}$ & 27 \\
\hline $1982-1985$ & 3 & Antioquia & 23 \\
\hline $1989-1998$ & 2 & Antioquia & 28 \\
\hline 1955 & 2 & $\mathrm{SD}$ & 9 \\
\hline 1956 & 1 & SD & 29 \\
\hline $1962-1963$ & 25 & SD & 30 \\
\hline 1965 & 15 & SD & 31 \\
\hline
\end{tabular}

SD: $\sin$ dato 
Cuadro 3. Paracoccidioidomicosis y su distribución según departamento: definición de áreas endémicas de acuerdo con número de casos, por orden de frecuencia (1949-1999).

\begin{tabular}{lc}
\hline Departamento & Casos (n) \\
\hline Antioquia & 311 \\
Santander & 271 \\
Cundinamarca, Bogotá & 146 \\
Meta & 95 \\
Norte de Santander & 70 \\
Caldas & 47 \\
Boyacá & 39 \\
Tolima & 37 \\
Magdalena & 32 \\
Valle & 26 \\
Huila & 18 \\
Arauca & 16 \\
Cesar & 14 \\
Caquetá & 10 \\
Quindio & 9 \\
Risaralda, Bolívar, Guaviare & 7 \\
Córdoba, Chocó, Casanare & 4 \\
Cauca, Nariño & 3 \\
Atlántico, Guainía, Putumayo, Vaupés, Sucre & 2 \\
Amazonas & 1 \\
Vichada, San Andrés, La Guajira & 0 \\
\hline
\end{tabular}

Análisis realizado: mediana: 7 casos; percentil 25: 3 casos; percentil 75: 32 casos

diagnosticado casos. El análisis estadístico estableció una mediana de 4 años de diagnóstico para cada uno de ellos. En el cuartil 4, se agruparon ocho departamentos en los que la micosis había sido diagnosticada por más de 13 años; en el cuartil 3, nueve departamentos que la habían diagnosticado por 4 a 11 años, y en el cuartil 2, cinco departamentos que solamente lo habían hecho por 2 a 3 años. Con base en estos parámetros, se estableció que los primeros 8 departamentos eran endémicos, 14 eran de baja endemicidad, mientras que los 10 restantes fueron no endémicos (cuadro 4).

El empleo de ambos análisis permitió colocar a 9 departamentos en la categoría de endémicos puesto que en ellos coincidieron ambos análisis, a saber: Antioquia, Santander, Cundinamarca, Meta, Norte de Santander, Caldas, Boyacá, Tolima y Magdalena (figura 1).

La tasa de incidencia obtenida con base en el número de casos de la micosis en los 9 departamentos designados como endémicos varió de 2,5 a $30 / 1^{\prime} 000.000$ hab (cuadro 5 ). La tasa nacional de incidencia/1'000.000 hab en 1973 y
Cuadro 4. Paracoccidioidomicosis y su distribución según departamento: definición de áreas endémicas de acuerdo con el número de años de diagnóstico (1980-1999).

\begin{tabular}{lc}
\hline Departamento & Número de años \\
\hline Antioquia & 20 \\
Santander & 19 \\
Cundinamarca, Bogotá & 18 \\
Meta, Norte de Santander & 16 \\
Caldas & 14 \\
Boyacá, Tolima & 13 \\
Magdalena, Huila & 11 \\
Arauca & 8 \\
Valle & 6 \\
Cesar & 7 \\
Guaviare, Quindío, Risaralda & 5 \\
Caquetá & 4 \\
Bolivar & 3 \\
Atlántico, Casanare, Nariño, Putumayo, & \\
Vaupés, Guanía & 2 \\
Amazonas, Sucre, Córdoba & 1 \\
Vichada, San Andrés, La Guajira, Cauca, Chocó & 0 \\
\hline
\end{tabular}

Análisis realizado: mediana: 4 años de casos notificados; percentil 25: 3 años; percentil 75: 13 años

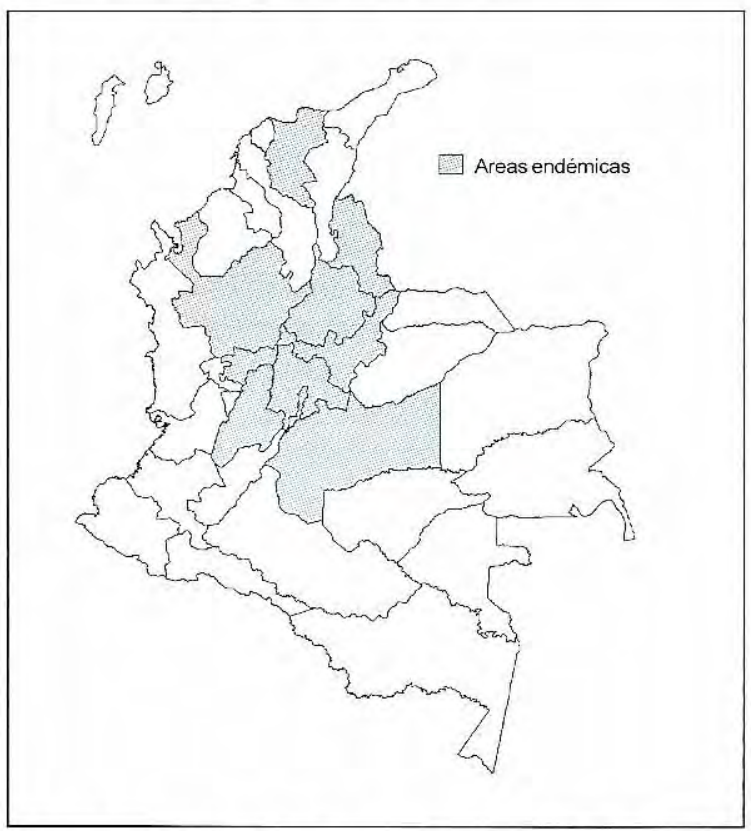

Figura 1. Definición de áreas endémicas de paracoccidioidomicosis con base en el número de casos (1949-1999) y el número de años de diagnóstico (1980-1999). Los departamentos coloreados corresponden a las áreas de mayor endemicidad.

de 1980 a 1995, está consignada en la figura 2; la tasa nacional máxima alcanzada durante los 19 años analizados fue de 2,4/1'000.000 hab en 1980. 
Cuadro 5. Paracoccidioidomicosis: tasas de incidencia (por millón de hab) por departamento de acuerdo con el número de habitantes (1984 y 1994).

\begin{tabular}{lcc}
\hline Departamento & $\begin{array}{c}\text { Tasa/10 } \\
\mathbf{1 9 8 4}\end{array}$ & $\begin{array}{c}\text { Tasa/10 } \\
\mathbf{1 9 9 4}\end{array}$ \\
\hline Antioquia & 2,6 & 1,6 \\
Santander & 4,4 & 2,2 \\
Cundinamarca & 5,0 & 0,1 \\
Meta & 29,0 & 6,4 \\
Norte de Santander & 7,3 & 1,7 \\
Caldas & 1,1 & 2,9 \\
Boyacá & 4,0 & 0,8 \\
Tolima & 0,8 & 0,8 \\
Magdalena & 3,3 & 0,9 \\
País & $\mathbf{2 , 2}$ & $\mathbf{0 , 5}$ \\
\hline
\end{tabular}

Ocho de los 9 departamentos endémicos se encuentran en la región andina colombiana y el restante en la Sierra Nevada de Santa Marta. Estos departamentos tienen pisos térmicos entre 1.000 y $2.000 \mathrm{~m}$ de altura sobre el nivel del mar, y corresponden a las regiones cafeteras colombianas.

\section{Discusión}

El presente estudio y los correspondientes análisis tuvieron como objetivo definir y establecer la condición de endemia de la paracoccidioidomicosis en los departamentos colombianos. Por definición, la enfermedad endémica es aquélla que se presenta constantemente en un área geográfica o en un grupo poblacional dado; igualmente, el término se aplica a la prevalencia usual de la enfermedad dentro de tal área o grupo (5).

Se considera que la paracoccidioidomicosis es la micosis sistémica de mayor prevalencia en Latinoamérica; no obstante, y puesto que no es una enfermedad de notificación obligatoria en los países afectados, la prevalencia real es desconocida (4, 32). Los datos disponibles señalan que $80 \%$ de los casos han sido descritos en Brasil, seguido a buena distancia por Colombia y Venezuela $(4,6,32)$. A pesar de los problemas inherentes a la falta de notificación obligatoria, la información suministrada en la literatura revela que la micosis se puede considerar endémica si cumple el requisito de presentarse constantemente en un área geográfica o en un grupo poblacional dado $(4,5)$.
Tasa $/ 10^{6}$

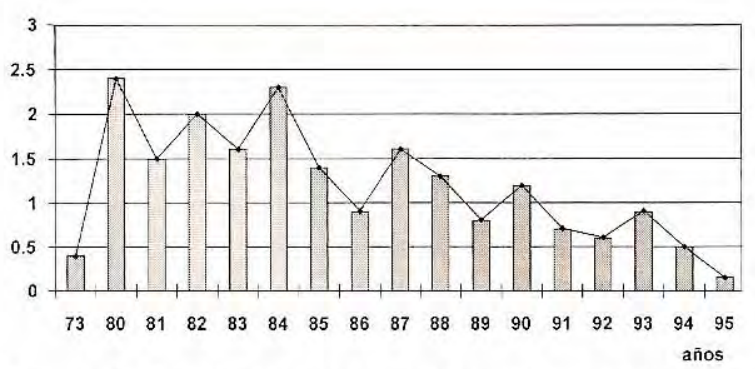

Figura 2. Paracoccidioidomicosis: tasa nacional de incidencia. 1973, 1980-1995, $n=667$

En nuestro país, con base en el número de casos diagnosticados se han considerado ciertas áreas como endémicas para la micosis. En este análisis, se pretendió confirmar la anterior consideración en una forma sistemática. Fue así como se emplearon métodos de análisis basados en la mediana y en los cuartiles, aplicados a dos datos obtenidos de las fuentes de información, a saber, el número de casos diagnosticados por departamento en los 50 años transcurridos desde que fuera informado el primer caso $(3-8,10-28)$, y el número de años en los que durante un período de 20 años, la entidad había sido diagnosticada por los departamentos. Ambos análisis coincidieron en señalar 8 de los 32 departamentos del país como endémicos, lo cual validó los resultados obtenidos. Es así como es posible conocer la distribución real de las áreas endémicas para esta micosis en Colombia. Como departamentos endémicos fueron identificados Antioquia, Santander, Cundinamarca, Meta, Norte de Santander, Caldas, Boyacá y Tolima, todos situados en la región centro-oriental andina del país, y Magdalena, localizado en la Sierra Nevada de Santa Marta.

Si se hubiese empleado solamente el análisis de las tasas de incidencia de la enfermedad en los 9 departamentos señalados como endémicos, ejercicio que se realizó con dos años $(1984,1994)$, los resultados no hubieran sido tan informativos. En efecto, se demostró que las tasas no eran un parámetro adecuado para definir la endemicidad ya que el análisis correspondiente señaló cómo en todos los departamentos, con la excepción de Caldas, las tasas habían disminuido. Sería 
interesante estudiar las posibles causas de esta disminución en nuestro país. La tasa nacional de incidencia osciló entre 0,1 y 2,4/1'000.000 hab y la tasa mas baja fue la de 1995 . Como ya se señaló, las causas de la disminución de las tasas debe ser explorada.

Es un reto tratar de definir el nivel de endemicidad de una enfermedad sin tener fuentes fiables de información interna y externa. Normalmente, se usan escalas empleadas en otros países o largamente estudiadas. En este caso, decidimos usar como patrón nuestra propia distribución de casos para identificar las áreas de mayor circulación del hongo en el país, haciendo comparaciones con nuestros propios patrones.

Si se tomara como ejemplo a Brasil, país donde se consideran como de alta endemia aquellas regiones donde la incidencia anual oscila alrededor de 1 a 3 casos por 100.000 hab $(4,32,33)$, los datos correspondientes a Colombia no permitirían calificar nuestros departamentos como de alta endemicidad, puesto que las incidencias fueron menores. Sin embargo, debe recordarse que Brasil cuenta con más de $60 \%$ de todos los casos informados en el área endémica (4).

Se conoce que la distribución de la micosis es heterogénea, ya que se describen áreas que cuentan con un número importante de casos y que están cerca de otras donde la micosis no ha sido diagnosticada $(4,6,32)$. En el análisis de los 1.191 casos diagnosticados en un período de 50 años (1949-1999) (3-8,10-28), se confirmó que entre nosotros la distribución de la micosis era también heterogénea.

La información suministrada por el presente análisis también señala las regiones que por su endemicidad serían más adecuadas para la búsqueda del hábitat natural de $P$. brasiliensis. Hasta el presente, se conoce que existe otro hospedero para el hongo, el armadillo (33) y, de hecho, en un estudio reciente realizado en el departamento de Caldas, se tuvo en cuenta la reconocida endemicidad de esta región para buscar allí armadillos infectados por el hongo, encontrándose positivo uno de ellos (34). Este hallazgo valida la información suministrada en el presente estudio.
La clasificación de áreas endémicas aquí presentada permitirá, si bien indirectamente, detectar cualquier cambio futuro que se presente en el diagnóstico de la micosis, como ocurrió en la región amazónica de Brasil, área no considerada como endémica, pero en la cual, en época reciente, se han diagnosticado varios pacientes (6).

\section{Agradecimientos}

A Jenny Hernández, estudiante de bacteriología, por su gran ayuda en la consecución de la información durante su rotación en el Grupo de Microbiología del INS; al doctor Gerzaín Rodríguez, coordinador del Grupo de Patología del INS, por permitirnos revisar los archivos de su Grupo, y a los doctores Myrtha Arango, Angela María Tobón, David Santiago Rosero y Daniel Alberto Calle, por compartir con nosotros la información recopilada en los archivos de la CIB y en los del Laboratorio de Patología del Hospital Universitario San Vicente de Paúl de Medellín. Igualmente, manifestamos nuestro aprecio a las profesoras Marta Rincón y María Inés Alvarez por algunos de los datos de Santander y del Valle del Cauca.

\section{Referencias}

1. Greer DL, Restrepo A. The epidemiology of paracoccidioidomicosis. En: The epidemiology of human mycotic diseases. AL-Doory Y, editor. Chicago, Illinois, USA: Charles Thomas Publisher; 1975. p.117-41.

2. Lacaz CS. Historical evolution of the knowledge on paracoccidioidomycosis and its etiologic agent, Paracoccidioides brasiliensis. En: Franco $M$, da Silva Lacaz C, Restrepo A, Del Negro G, editores. Paracoccidioidomycosis. Boca Ratón, Fla, USA: CRC Press; 1994. p.1-7.

3. Méndez Lemaitre A. Blastomicosis suramericana y otras micosis en Colombia. Revista del Hospital de La Samaritana 1950;1:3-13.

4. Wanke B, Londero AT. Epidemiology and paracoccidioidomycosis infection. En: Franco M, da Silva Lacaz C, Restrepo A, Del Negro G, editores. Paracoccidioidomycosis. Boca Ratón, Fla, USA: CRC Press; 1994. p.109-15.

5. Lilienfield A. Fundamentos de Epidemiología. México: Interamericana; 1979.

6. Wanke B. Epidemiology of paracoccidioidomycosis: an emerging health problem in the Brazilian Amazon region. VII Encontro Internacional sobre paracoccidioidodomicose. Campos de Jordâo, Sâo Paulo, 1999. MR-03 
7. Restrepo A. Ecology of Paracoccidioides brasiliensis. En: Franco M, da Silva Lacaz C, Restrepo A, Del Negro $\mathrm{G}$, editores. Paracoccidioidomicosis. Boca Ratón, Fla, USA: CRC Press; 1994. p.121-8.

8. Londoño R, Blair J. Comentarios sobre un caso de blastomicosis suramericana. Anot Ped 1954;1:153-61.

9. González P. Blastomicosis suramericana. Salubridad 1956;1: $55-6$.

10. Londoño F. La blastomicosis suramericana en Colombia Rev Fac Med Univ Nal 1957;25:101-18.

11. Retamoso BM. Blastomicosis suramericana. Rev Soc Med Quirur Atlántico 1963;9:91-3.

12. Alvarez R. La blastomicosis suramericana en el Valle del Cauca. Ant Med 1965;15:243-6.

13. Borrero J, Restrepo A, Robledo M. Blastomicosis suramericana de forma pulmonar pura. Ant Med 1965; 15:503-16.

14. Saravia J, Rocha H, Argüello M. Aspectos clínicos y de laboratorio de la blastomicosis suramericana. Rev Fac Med Univ Nac 1965;33:189-204.

15. Barrientos MA, Robledo M. Blastomicosis en la laringe. Acta Otorrinolaringológica de Colombia 1966;1:8-17.

16. Restrepo A, Moncada LH. Comportamiento inmunológico de veinte pacientes con paracoccidioidomicosis. Ant Med 1967;17:211-31.

17. Restrepo A, Espinal LS. Algunas consideraciones ecológicas sobre la paracoccidioidomicosis en Colombia. Ant Med 1968;18:433-46.

18. Restrepo A, Robledo M, Gutiérrez F, Sanclemente M, Castañeda E, Calle G. Paracoccidioidomycosis (South American Blastomicosis): a study of 39 cases observed in Medellín, Colombia. Am J Trop Med Hyg 1970; 4:68-76.

19. Saravia J, Restrepo M, Toro G, Vergara I. Paracoccidioidomicosis del sistema nervioso central. Rev Fac Med Univ Nal 1973;39:27-37.

20. Díaz O. Paracoccidioidomicosis diseminada con manifestación genital y perineal. Informe de un caso. Ant Med 1975;25:65-9.

21. Castañeda E, Ordóñez N, Bustos L, Sánchez J. Paracoccidioidomicosis: diagnóstico por el laboratorio de 87 casos. Acta Med Colomb 1981;6:339-48.

22. Robledo J, Restrepo A. Paracoccidioidomicosis y tuberculosis. Rev Medicina UPB 1984;3:49-54.
23. González A, Naranjo MS. El caso de infecciosas: micosis sistémicas severas y su tratamiento con derivados imidazólicos. Rev Medicina UPB 1987;6:4755.

24. Rincón M. Paracoccidioidomicosis: informe de 138 casos diagnosticados en Santander. Rev UIS-Salud 1989;17:51-61.

25. Spir N. Paracoccidioidomicosis: revisión retrospectiva de 100 casos. Rev CES Med 1991;5:125-30.

26. Villar LA, Gómez A, Rincón M, Olarte E, Bárcenas C, Silva FA. Paracoccidioidomicosis en Santander: aspectos clínico-epidemiológicos(1953-1992). Médicas UIS1992;6:182-8.

27. Castillo J, Ordóñez N, López S, Castañeda E, Grupo del Programa de Micosis Pulmonares. Paracoccidioidomicosis: diagnóstico por el laboratorio de 333 casos. Biomédica 1994;14:230-9.

28. Tobón A, Orozco B, Estrada S, Jaramillo E, De Bedout C, Arango M, Restrepo A. Paracoccidioidomycosis and AIDS: report of the first two Colombian cases. Rev Inst Med Trop Sao Paulo 1998;40:377-81.

29. Dueñas V, García C, Ramírez J. Contribución al estudio de la blastomicosis suramericana en Colombia. An Soc Biol Bogotá 1955;7:1-19.

30. Restrepo A, Calle G, Sánchez J, Correa A. A review of medical mycology in Colombia, S.A. Mycopathol Mycol Appl 1962;12:93-102.

31. López H, Hurtado H, Correa G. La micosis profunda en el Hospital San Juan de Dios. El Médico 1965;10:20-7.

32. Franco M, Mendes RP, Moscardi-Bacchi M, Rezkallah-Iwasso M, Montenegro MR. Paracoccidioidomycosis. Bailliere's Clin Trop Med Comm Dis 1989; 4:185-220.

33. Restrepo A. Ecology of Paracoccidioides brasiliensis. En: Franco M, da Silva Lacaz C, Restrepo A, Del Negro G, editores. Paracoccidioidomycosis. Boca Ratón, Fla, USA: CRC Press; 1994. p.121-30.

34. Corredor GC, Castaño JH, Peralta LA, Díez S, Arango M, McEwen J, Restrepo A. Isolation of Paracoccidioides brasiliensis from the nine-banded armadillo Dasypus novemcinctus, in an endemic area for paracoccidioido-mycosis in Colombia. Rev Iberoam Micol 1999;16:216-20. 